\title{
Le Capital Immatériel : Evaluation Et Importance Cas Des Entreprises Marocaines Cotées En Bourse
}

\author{
Omar Taouab \\ Professeur Habilité à l'école nationale de commerce et de gestion de Kénitra \\ Lotfi Benazzou \\ Professeur à l'école nationale de commerce et de gestion de Kénitra \\ Aziz babounia \\ Professeur Habilité à la faculté Polydisciplinaire de Tétouane
}

doi: 10.19044/esj.2016.v12n10p304 URL:http://dx.doi.org/10.19044/esj.2016.v12n10p304

\begin{abstract}
Currently, we live in an economy that has changed considerably since the 1990s. This is the time where the economies have moved from the industrial to the information age. For about a decade, companies have switched to the immaterial economy in addition to the physical value (fixed assets: buildings, workshops, computers, vehicles, etc.) and the cash value (cash, receivables, etc.).There is a gaseous value, which is the intangible capital. This new notable concept is the subject of this research, which is to calculate the intangible capital of a sample of listed Moroccan companies, according to an approximate approach, based on publicly available data on Casablanca Stock Exchange website. The purpose of this research is to answer the following questions: What is intellectual capital? What are the components of intangible capital? And what is the weight of intangible capital in Moroccan companies?
\end{abstract}

Keywords: Intangible assets, human capital, Structural Capital, Organizational Capital, Goodwill, Intellectual Capital, Brand equity

\section{Résumé}

Nous vivons dans une économie qui a considérablement changé depuis les années 90, c'est à ce temps que les économies sont passées de l'ère industrielle vers l'ère de l'information. Depuis un peu plus d'une décennie, les entreprises ont basculé dans une économie à dominante immatérielle. Ainsi, et en plus de la valeur solide (immobilisation : immeubles, ateliers, ordinateurs, véhicules, etc.) et la valeur liquide (trésorerie, créances, etc.), il existe une valeur gazeuse qui est le capital immatériel. Cette nouvelle notion notable fait l'objet de cet article, qui consiste à exposer le capital immatériel 
d'un échantillon de sociétés marocaines cotées en bourse, selon une approche approximative, en se basant sur les données publiquement disponibles sur le site de la bourse de Casablanca. L’objet de cette recherche est de répondre aux questions suivantes : Qu'est-ce que le capital immatériel ? Quelles sont les composantes du capital immatériel ? Et quel est le poids du capital immatériel dans les entreprises Marocaines?

Mots clés: Capital immatériel, actifs incorporels, capital humain, Capital structurel, Capital organisationnel, Goodwill, Capital intellectuel

\section{Introduction}

L'essor des Nouvelles Technologies de l'Information et de la Communication (NTIC) a consacré l'entrée des sociétés globalisées dans une nouvelle ère : la nouvelle économie et l'ordinateur, son porte-drapeau érigé en prolongement direct du cerveau, supplante l'économie industrielle et marque l'arrêt du rôle moteur du muscle dans le fonctionnement de la machine à vapeur.

On assiste, en effet, au passage d'une économie fondée sur l'effort physique, relevant du domaine tangible, à celle diamétralement opposée du savoir et de la connaissance, relevant du domaine intangible. Ce changement se traduit par des flux d'informations continus et instantanés, dans un contexte de mondialisation des échanges, de globalisation de la finance et de tertiarisation des activités et des emplois. Cette profonde mutation est observable au cœur même des états financiers, ou l'on assiste à l'apparition de plus en plus accrue des actifs immatériels.

L'émergence de la nouvelle économie marque ainsi l'avènement de ce qu'il convient désormais de qualifier du « capital immatériel », " Goodwill », « Capital humain », « Capital intellectuel », autant de termes synonymes qui font référence à cette nouvelle notion comptable qui suscite la curiosité des économistes, analystes et financiers.

Les ressources immatérielles sont la face cachée de l'entreprise, elles sont le principal facteur de différenciation des entreprises et, en effet, peu prises en compte dans les états financiers. Or, elles représentent en moyenne les deux tiers du patrimoine d'une entreprise et sont aujourd'hui plus qu'hier à la source de sa compétitivité.

Il est donc primordial de prendre en compte cette richesse cachée lors de l'évaluation des entreprises, cette évaluation qui est au cœur de la finance.

Jusqu'à présent, l'immatériel était considéré, et donc traité, comptablement comme des charges et non pas des actifs. C'est le cas de la $\mathrm{R} \& \mathrm{D}$, des efforts de création, du capital humain, les marques, etc.

Créer une marque, par exemple, génère, en effet, des investissements particulièrement lourds : agence de communication, dépôt de brevet ou de 
protection. Des coûts qui auront un impact direct sur le compte de résultat mais qui n'apparaissent jamais au bilan.

Cette recherche, qui est en partie exploratoire et qui traite le problème d'évaluation et de gestion du capital immatériel des entreprises, aura comme objectif de répondre aux questions suivantes :

- $\quad$ Qu'est-ce que le capital immatériel ?

- Quelles sont les composantes du capital immatériel?

- A quoi consistent la notation et le calcul des différents actifs immatériels ?

La problématique de notre sujet est la suivant :

Quelle importance les entreprises marocaines cotées en bourse de Casablanca accordent-elles aux actifs immatériels? Leurs actifs immatériels ont-ils le même poids que leurs homoloques européens ?

Pour répondre à cette problématique nous avons procéder à calculer le poids, l'importance et l'évolution du capital immatériel pour un échantillon d'entreprises marocaines cotées en bourse de Casablanca dans le but d'exposer la valeur immatérielle.

Cette recherche porte sur une brève revue de la littérature spécialisée, ensuite la méthodologie du travail utilisée sera présentée, pour finir enfin par le dépouillement des résultats et leurs discussions.

\section{Revue de la littérature}

Il n'existe pas de définition du capital immatériel qui fassent l'unanimité parmi les experts et les financiers. Certains parlent du capital immatériel, alors que d'autres préfèrent employer le terme «capital intellectuel » ou «capital intangible ». Le nombre important de définitions pour désigner ce terme traduit la diversité des acteurs, comme les investisseurs, les organismes comptables, les universitaires, et les consultants, ainsi que leur intérêt particulier pour le traitement de la question. De plus, plusieurs chercheurs ne font pas la différence entre les notions voisines au capital immatériel, d'où l'importance de définir le contour des terminologies employées.

Avant de définir sous un angle pratique le capital immatériel, il est préférable de pouvoir différencier les actifs immatériels de leurs cousins incorporels qui ont une filiation plus comptable et employés souvent comme leurs synonymes. 


\section{Les actifs intangibles ou actifs incorporels}

Les actifs intangibles et incorporels sont définis par la norme comptable internationale IAS $38^{1}$ comme étant « un actif non monétaire identifiable sans substance physique, détenu en vue de son utilisation pour la production ou la fourniture de biens ou de services, pour une location à des tiers ou à des fins administratives... Un actif incorporel est une ressource contrôlée par une entreprise du fait d'évènements passés et dont des avantages économiques futurs sont attendus. »

Baruch Lev (2002) ${ }^{2}$ définit les actifs incorporels comme les" knowledge assets» (traduit par les actifs issus du savoir, de l'information ou de la connaissance) et les classe en 4 catégories :

- Innovation, recherche et développement,

- $\quad$ Notoriété de la marque, image de marque : cela permet de considérer un produit ou un service différent de la concurrence et de le vendre plus cher sur le marché. .

- $\quad$ Actifs structurels: ce ne sont pas des inventions ni des innovations fortes, mais des compétences permettant de travailler mieux.

- Monopole ou oligopole : ce sont les barrières à l'entrée pour les compétiteurs. Elles peuvent être légales comme les licences d'exploitation d'un opérateur Internet. La position de leader du marché procure souvent un avantage concurrentiel puissant qui représente un actif incorporel facile à déterminer.

L'actif incorporel qui a suscité le plus d'études est le fond de commerce ou le «goodwill» que l'on appelle aussi achalandage. La traduction française de "goodwill »est la «survaleur ", ou " écart d'acquisition », ce qui correspond mieux à sa signification réelle que le terme américain qui fait explicitement référence à la relation entre la firme et sa clientèle, à sa réputation. Cette survaleur n'est que le solde qui sépare la valeur réelle de la firme de la somme des actifs qu'elle possède au moment de son acquisition. Cette composante comporte généralement plusieurs éléments incorporels qui n'ont pas pu être comptabilisés de façon distincte. En effet, il s'agit de ressources que l'on ne parvient ni à définir, ni à isoler, encore moins à mesurer.

\section{Le capital intellectuel}

Le capital intellectuel est un terme qui a été conçu par les experts en ressources humaines afin de mettre de l'avant l'importance du facteur humain

\footnotetext{
${ }^{1}$ IAS 38 est une norme issue du référentiel comptable international IFRS et relative aux immobilisations incorporelle

${ }^{2}$ Lev. B (2002). Rethinking accounting. Financial Executive. Morristown, 18 (2), p. $34-38$
} 
dans la valeur des entreprises (J- Pépin, 2006) ${ }^{3}$. Ce capital est étroitement associé au « knowledge management» (la gestion des connaissances). Il est composé de l'ensemble des connaissances du personnel et de toutes autres ressources intellectuelles acquises par l'expérience ou l'apprentissage en vue d'être utilisé dans le but de la création de la richesse (Hussi 2004) ${ }^{4}$.

Selon Moon et $\mathbf{K y m}^{\mathbf{5}}$ le capital intellectuel est la combinaison du capital humain et du capital structurel. Le capital humain est composé des connaissances, compétences et aptitudes des employés à apporter des solutions aux clients; le capital structurel est composé de tout ce qui reste quand les employés rentrent chez eux (base de données, fiches clients, software, manuels, études de marchés, structures organisationnelles.

\section{Le capital immatériel}

Une nouvelle discipline naissait en Suède baptisée science de mesure de la valeur économique «intangible». Les auteurs ayant publié sur le capital immatériel sont nombreux et nous ne les citerons pas tous. La définition des actifs immatériels qu'ils proposent et leur identification est variable de l'un à l'autre et, à ce jour, aucun standard international n'émerge encore dans ce domaine.

Dans leur livre, le capital immatériel de l'entreprise, Michael Malone et Leif Edvinsson ${ }^{6}$ ne proposent pas de définition précise de la notion d'actifs immatériels, mais présentent une décomposition de la valeur de l'entreprise en une cascade d'actifs matériels et immatériels qui a valeur de définition ${ }^{7}$.

Selon Christian Pierrat ${ }^{8}$, un actif immatériel (ou incorporel) est un élément du patrimoine de l'entreprise qui présente simultanément plusieurs caractéristiques :

- $\quad$ absence de substance physique;

- $\quad$ durée de vie indéterminée;

- $\quad$ unicité ou, au moins, forte spécificité;

- $\quad$ grande incertitude sur ses revenus futurs;

\footnotetext{
3 - François Pépin. Capital immatériel, 7 jours pour comprendre, journées d'étude. CIGREF 2006

${ }^{4}$ Huss.T(2004): Journal of Knowledge Management (page 44)

${ }^{5}$ Yun Ji Moon and Hyo Gun Kym: A Model for the Value of Intellectual Capital (2006) (page 258)

${ }^{6}$ Michael Malone et Leif Edvinsson (1999); le capital immatériel de l'entreprise. éd Maxima

7 Leif Edvinsson était l'un des dirigeants, à l'époque, de la compagnie d'assurance Skandia, pour laquelle il mit au point un modèle de mesure de la valeur immatérielle de son entreprise, le «Navigateur».

${ }^{8}$ Christian Pierrat, Immatériel et comptabilité, in Encyclopédie de Comptabilité, Contrôle de gestion et Audit, éd. Economica
} 
- $\quad$ difficilement séparable des autres actifs.

Selon Alan Fustec et Bernard Marois ${ }^{9}$, un actif immatériel est un constituant de l'entreprise, identifiable séparément, qui participe aux opérations génératrices de rentabilité présente ou future, mais dont la valeur ne figure pas au bilan.

Par extension, des actifs matériels visibles au bilan peuvent être porteurs d'une valeur immatérielle ou d'un goodwill invisible. Ainsi peut- on arguer que le capital immatériel est partout. Dès lors qu'un élément de l'entreprise a une valeur supérieure à son coût, il est porteur de valeur immatérielle.

\section{Les composantes du capital immatériel}

Les actifs immatériels (ni matériels, ni financiers) sont une formidable source d'énergie pour se différencier dans un monde hyperconcurrentiel et source de singularité et sont difficilement comparables d'une entreprise à l'autre, selon Jérome JULIA ${ }^{10}$.

Ils se rangent en trois catégories :

$\checkmark \quad$ Le capital humain recouvre les compétences, l'expérience collective, les hommes clés (ce qui est dans la tête des employés de l'entreprise);

$\checkmark \quad$ Le capital organisationnel ou structurel comprend les brevets, procédures, système d'information, bases de données, valeurs... (ce qui reste dans l'entreprise à la fin de la journée);

$\checkmark \quad$ Le capital relationnel enfin, regroupe les clients, fournisseurs, réseaux, actionnaires, régulateurs... (ce qui relie l'organisation à son environnement).

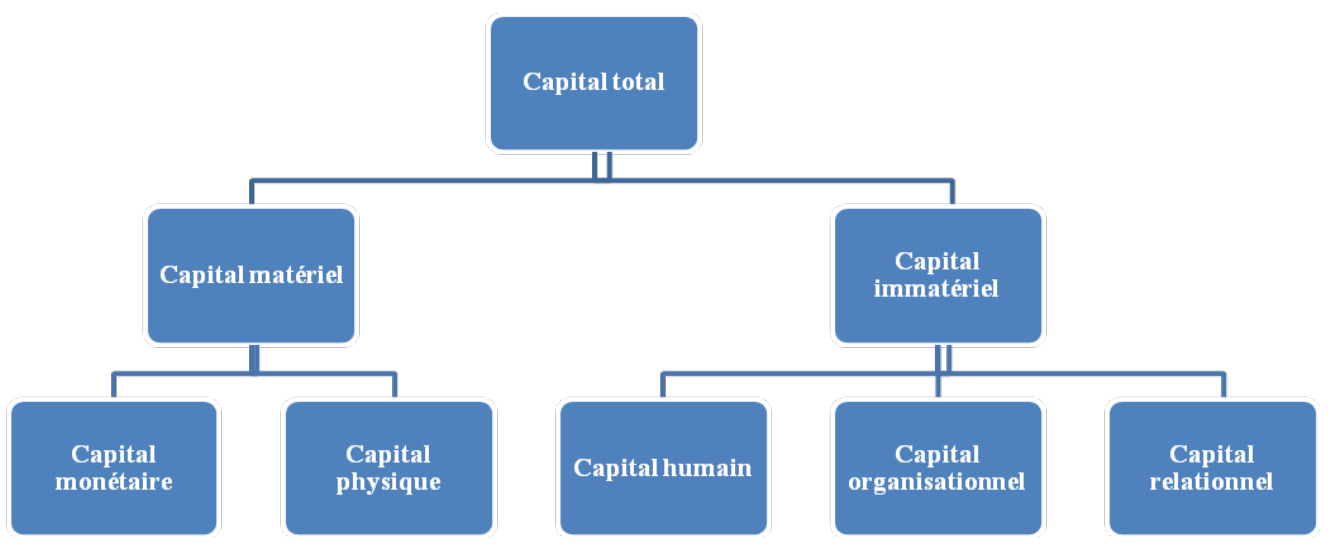

Figure 1 : Capital immatériel - 7 jours pour comprendre, publié par CIGREF P : 31

19, éd d'organisation

10 Jérome JULIA : Président de l’Observatoire de l’immatériel, Directeur associé au sein du cabinet de Stratégie et Management Kea \& Partners 


\section{Méthodologie de travail}

Suivant une méthodologie du fameux cabinet Ernest \& Young d'audit comptable et financier, l'un des Big Four et le troisième réseau mondial en termes de chiffre d'affaires, qui a mené une étude sur le capital immatériel sur un échantillon composé d'une centaine d'entreprises européennes cotées en bouse, nous avons procédé de la même façon à calculer le poids, l'importance et l’évolution du capital immatériel pour un échantillon d'entreprises marocaines cotées en bourse de Casablanca.

Pour mener cette étude, il était nécessaire de passer par la bourse de Casablanca, qui nous était la seule source de collecte des données publiquement disponibles. Notre échantillon comprend plus d'une trentaine de grandes sociétés exerçant dans plusieurs secteurs: immobilier, BTP, distribution, etc. Cela nous a permis de dessiner une vision globale, de comparer les entreprises du même secteur et de comparer les secteurs entre eux.

La valeur de l'immatériel de chaque entreprise est en effet obtenue par la différence entre d'une part la valeur de l'entreprise (VE) à partir de la capitalisation boursière (CB) et ses dettes financières (DF) à la même date, et d'autre part, la valeur comptable des actifs figurant au bilan:

\section{Capitalisation Boursière + Dettes financières = Actifs économiques + Capital immatériel}

Cette approche constitue une approximation dans la mesure où cela revient à considérer que l’intégralité de l’écart entre la valeur de marché des entreprises et la valeur des éléments comptables correspond à de l'immatériel, sans toutefois oublier de préciser que les éléments de revalorisation des autres types d'actifs (corporels) sont non-significatifs.

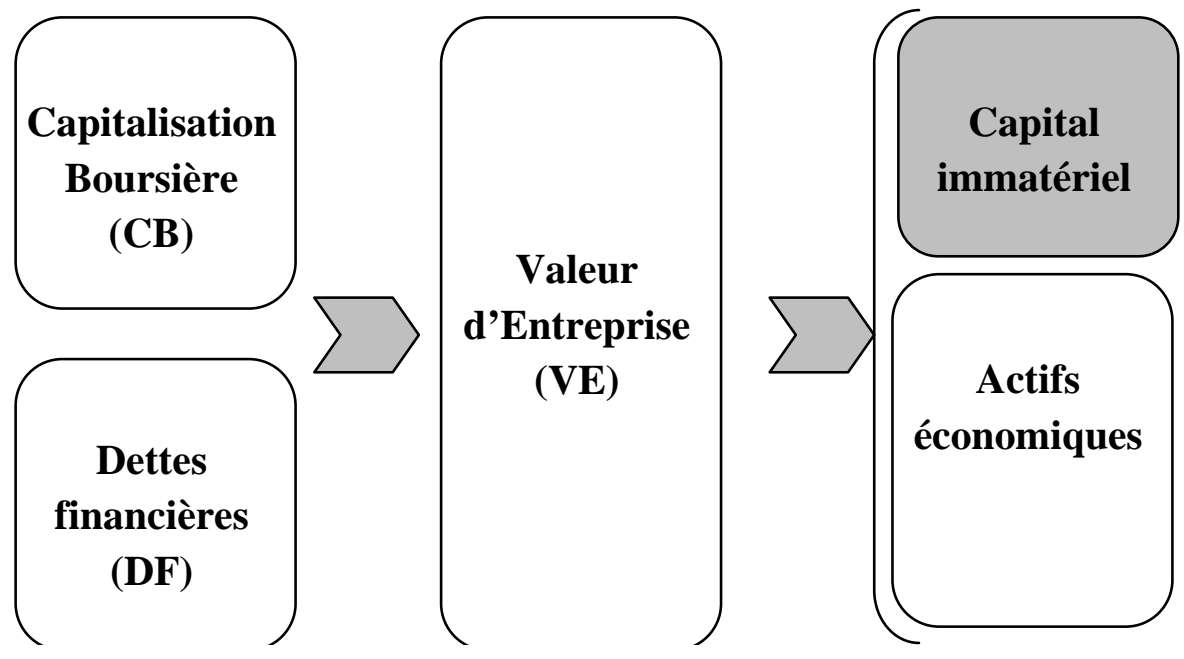

Figure 2 : Estimation de la valeur de l’immatériel 


\section{Résultats et Discussions}

\subsection{Les tendances sectorielles} entre 2012 et 2014

Après la constitution de la base de données, et l'établissement des rapports, nous avons abouti aux résultats ventilés ci-après :

- Le secteur de télécommunication représenté par Itissalat-Al-Maghreb, qui est la première société de télécommunication au Maroc, détient une part de $54 \%$ de la valeur des actifs immatériels de notre échantillon. Cela est expliqué par la nature des services offerts, sa grande taille, et le fait qu'elle est la seule représentante du secteur de la télécommunication qui est fortement dotée du capital immatériel.

- Une légère variation entre 2012 et 2013, quelques secteurs ont pu développer leur capital immatériel au détriment des autres, le leader en télécommunication maintient toujours sa position, tandis que d'autres secteurs comme le secteur de l'immobilier qui s'est détérioré à cause de la conjoncture. Le secteur du matériel, logiciels et services
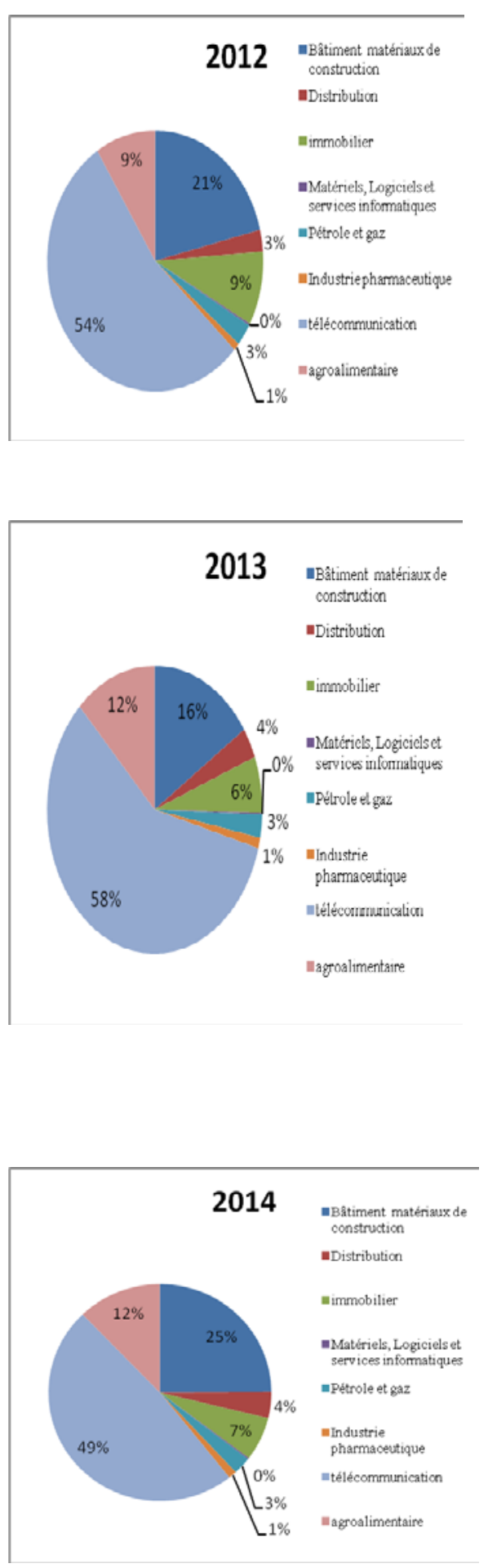


\section{Dépouillement des résultats}

L'échantillon, composé de 30 entreprises marocaines cotées en bourse exerçant dans 8 secteurs d'activités différents, fait ressortir les résultats suivants :

$\checkmark \quad$ La part de l'immatériel représente en moyenne plus de $50 \%$ de leur valeur totale.

$\checkmark \quad$ Parmi les secteurs fortement doté du capital immatériel il y a le secteur de télécommunication, agroalimentaire, pharmaceutique et matériels, logiciel et service informatique.

$\checkmark \quad$ En moyenne, 23\% de la valeur de l'immatériel est comptabilisée au bilan en 2012, contre 29\% en 2014.

$\checkmark \quad$ L'immatériel est passé en moyenne de 52,31\% en 2012 à 55.49\% de leur valeur en 2014.

$\checkmark \quad$ Le secteur du bâtiment et matériaux de construction s'est dégradé en 2013 à cause d'une chute remarquable de ces actifs immatériels.

Notons que les variations constatées sont dues à plusieurs facteurs, qu'on ne peut pas cerner d'une façon absolue, mais parmi les variables qui peuvent expliquer ces variations, nous pouvons citer la valeur de marché de capitaux propres qui résulte de la multiplication du nombre d'actions composant le capital de la société par le cours de bourse. Lorsque ce dernier chute, suite à la confrontation de l'offre et de la demande, la valeur du capital immatériel diminue aussi. C'est le cas du secteur des Bâtiments et matériaux de construction qui a vu son capital immatériel déprécié en 2013 d’une façon remarquable, presque toutes les entreprises de ce secteur ont perdu une fraction de leur valeur immatérielle suite à une dépréciation de leur cours de bourse.

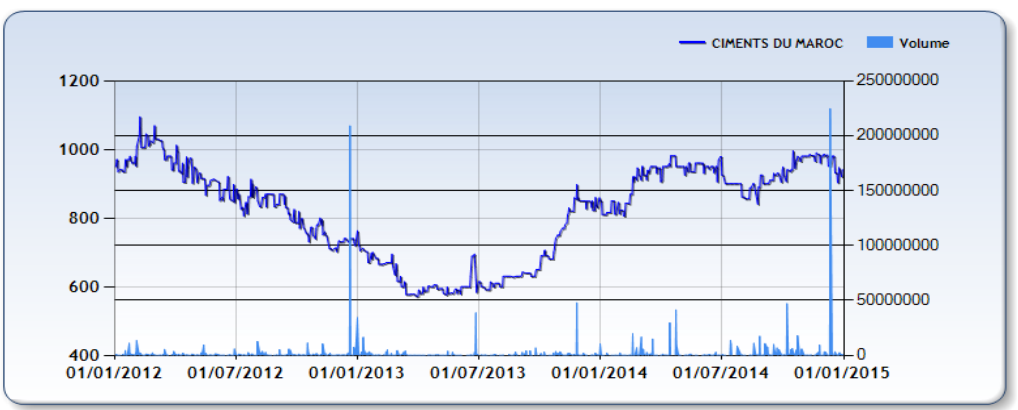

Figure 4 : Historique du cours boursier de Ciments du Maroc entre 2012 et 2014

\section{Conclusion}

Les objectifs de cette recherche consistaient à comprendre les actifs immatériels, comment ils sont identifiés et mesurés et quelle valeur les entreprises marocaines accordent-elles aux actifs immatériels. 
Le capital immatériel ou patrimoine immatériel est un élément non monétaire et sans substance physique, constitué par les informations et connaissances détenues, et ayant une valeur positive, pour une organisation.

Il intègre ainsi le concept de capital-savoir en gestion des connaissances et il est reconnu aujourd'hui comme le levier de création de valeur majeur dans toutes les entreprises.

Cette recherche confirme que l'immatériel constitue bien la première richesse de l'entreprise. Cette richesse gazeuse suscite l'appétit des analystes, financiers et directeurs d'aujourd'hui même si elle ne fait pas l'objet d'un accord unanime. Cette notion naissante en Suède, est apparut pour traiter des synergies qui peuvent naître des interactions dans l'entreprise.

Notre problématique était de saisir l'importance et le poids du capital immatériel dans les entreprises marocaines cotées en bourse et de le comparer par rapport aux résultats de l'étude européenne, selon la même démarche du Fameux cabinet d'audit financier Ernest \& Young. Cette approche constitue une approximation dans la mesure où cela revient à considérer que l'intégralité de l'écart entre la valeur de marché des entreprises et la valeur des éléments comptables correspond à de l'immatériel, sans toutefois oublier de préciser que les éléments de revalorisation des autres types d'actifs (corporels) sont non-significatifs.

Les résultats ont montré que la richesse gazeuse a une moyenne qui dépasse $50 \%$ de la valeur totale de l'entreprise, et qui est en progression continue, sans oublier qu'aujourd'hui les entreprises prennent conscience de cette valeur cachée, vue que la part des immatériels comptabilisés au bilan ne cesse d'évoluer.

Les entreprises doivent franchir ce monde doté d'ambigüité, et essayer de bien appréhender leur capital immatériel, ses composants, ses leviers, tout en concevant des outils et des indicateurs de mesure pour mieux les piloter dans la perspective de créer de la valeur.

\section{References:}

AHMED BOUNFOUR (2006), capital immatériel, connaissance et performance, édition l'Harmattan.

ALAN FUSTEC BERNARD MAROIS (2006). Valoriser le capital immatériel de l'entreprise, édition d’organisation.

ANDRE GORZ (2003) l'immatériel. connaissance valeur et capital édition : Galilée

BESSIEUX. O ET WALLISER.E (2011). Le capital immatériel de l'entreprise, un défi pour les comptables et les managers.éd. EMS éditions. BOISSOLIER P., (1993): L’investissement immatériel : gestion et comptabil isation, édition De Boeck-Wesmael. 
CAPPELLETI, LAURENT, (2012). Le contrôle de gestion de l'immatériel, une nouvelle approche du capital humain, édition : Dunod.

EDVINSSON L ET MALONE M (1999); le capital immatériel de l'entreprise éd Maxima.

EDVINSSON.L\&MALONE M, (1997),Intellectual capital, realising your co mpany's true value by finding its hidden brainpower, harperBusiness, NY.

HERMEL, LAURENT, ALBERT, LOUPPE, (2002). Evaluation du capital client, édition : Afnour

HERVE BACULARD ET JEROME JULIO(2011) ; les actifs immatériels, le nouveau modèle de croissance. éditions du Cherche Midi.

KAPLAN R.S. et NORTON D.P, (2001) : Comment utiliser le tableau de bord prospectif. Editions d'organisation.

LIONEL ESCAFFRE ET PAUL VALENTIN NGOBO (2008), capital immatériel et performance dans l'entreprise, édition : Broché.

PIERRAT CHRISTIAN, Immatériel et comptabilité, in Encyclopédie de Comptabilité, Contrôle de gestion et Audit, éd. Economica.

PIERRAT C, MARTORY B, (1996) : Gestion de l'immatériel, édition NAT HAN.

SVEIBY K-E., (1998),

Measuring Intangibles and Intellectual Capital - An Emerging

First Standard.

SVEIBY, K. E. (1997). La nouvelle richesse des entreprises: Savoir tirer profit des actifs immatériels de sa société. Éditions : Maxima.

VIOT CATHERINE, (2004). Le capital- marque : concept, mesure et valorisation,

Éd : e-thèque. 